\title{
Structure and Elasticity of Lipid Membranes with Genistein and Daidzein Bioflavinoids Using X-ray Scattering and MD Simulations
}

\author{
Mohit Raghunathan, ${ }^{\dagger, \S}$ Yuriy Zubovski, ${ }^{\dagger, \perp}$ Richard M. Venable, ${ }^{\ddagger}$ Richard W. Pastor, John F. Nagle, ${ }^{\dagger}$ \\ and Stephanie Tristram-Nagle*,†

\begin{abstract}
${ }^{\dagger}$ Biological Physics Group, Physics Department, Carnegie Mellon University, 5000 Forbes Avenue, Pittsburgh, Pennsylvania 15213, United States
\end{abstract} \\ ${ }^{\ddagger}$ Laboratory of Computational Biology, National Heart, Lung and Blood Institute, 5635 Fishers Lane, T-900 Suite, Rockville, \\ Maryland 20852, United States
}

\section{Supporting Information}

ABSTRACT: This work reports the effects of the bioflavinoids genistein and daidzein on lipid bilayers as determined by volume measurements, X-ray scattering, and molecular dynamics simulations. The experimental and simulated total molecular volumes were found to be in outstanding agreement with each other before the addition of genistein and daidzein and also after their addition. Both bioflavinoids inserted into the hydrocarbon region of both DOPC and diphytanoylPC near the carbonyls of the lipids and both decreased the bilayer thicknesses. The long axes of both bioflavinoids were oriented nearly parallel to the plane of the bilayer with their carbonyl groups preferentially pointed toward the proximal surface. A difference is that daidzein had a solubility limit of $\sim 0.14 \mathrm{~mol}$ fraction in DOPC ( $\sim .12 \mathrm{~mol}$ fraction in diphytanoylPC), whereas genistein was soluble at least to 0.20 mol fraction in both lipid membranes. Measurements of bending modulus $K_{\mathrm{C}}$ and simulation results for area compressibility modulus $K_{\mathrm{A}}$ indicate that both bioflavinoids soften bilayers.

\section{INTRODUCTION}

Ion-channel modifiers are generally thought to regulate protein phosphorylation and dephosphorylation. One class of modifiers, the bioflavinoids, includes the well-studied genistein. ${ }^{1,2}$ For the cystic fibrosis transmembrane conductance regulator (CFTR) channel, genistein affects the wild-type channel and activates a mutant channel as a result of a change in its specific binding site. ${ }^{3}$ As a tyrosine kinase inhibitor, genistein could serve as an antitumor agent, since kinase activity is strongly correlated with the ability of retroviruses to transform cells. ${ }^{4-6}$ Another specific effect of genistein is to mimic the action of estrogen. ${ }^{7,8}$ Most estrogen effects are mediated by estrogen receptors, which are transcriptional regulators, but some are mediated by membrane receptors linked to calcium metabolism., ${ }^{9}$ The roles of estrogen; genistein; and another bioflavinoid, daidzein, on murine osteoclasts were investigated; estrogen > genistein > daidzein all reduced osteoclast differentiation, which may protect bone. ${ }^{11}$

In addition to these specific effects that require binding to proteins, bioflavinoids have also been reported to modulate ionchannel activity in a nonspecific way, that is, by altering the properties of the lipid membrane surrounding the channel. ${ }^{12,13}$ By changing the length of a gramicidin A $(\mathrm{gA})$ channel $^{12}$ and by using lipid membranes of varying thicknesses, ${ }^{13}$ the importance of hydrophobic mismatch in limiting both the onset and the lifetime of gA channel formation was demonstrated. It was suggested that genistein shifts the equilibrium from nonconducting monomers to conducting gA dimers by compensating for hydrophobic mismatch. This conclusion was reached because the magnitude of the effect of genistein increased with increasing hydrophobic mismatch between the channel length and the membrane thickness. ${ }^{13}$ It was further hypothesized that genistein affects protein-lipid coupling by changing the elastic properties of the membrane, which involve the lipid area compressibility modulus, $K_{\mathrm{A}}$ and the bending modulus, $K_{\mathrm{C}}$. An interesting contrast is that in $\mathrm{DPhyPC} / n$-decane black lipid membranes, daidzein only increased gA channel lifetimes half as much as genistein. ${ }^{13,14}$ Another reported difference between these two bioflavinoids is that daidzein, but not genistein, was reported to aggregate liposomes. ${ }^{15}$

In the present investigation, we use $\mathrm{X}$-ray diffuse scattering to measure $K_{\mathrm{C}}$ and to provide basic structural data for the effect of genistein and daidzein in DOPC and DPhyPC membranes. These data are used to validate molecular dynamics (MD) simulations, which then provide the area/unit cell of DOPC with bioflavinoids at 20 and $14 \mathrm{~mol} \%$, and the bioflavinoid

Received: December 10, 2011

Revised: February 1, 2012

Published: February 10, 2012 
positions in the DOPC membrane. In addition, we measure the molecular volume and use a volume-conserving fit of our diffuse X-ray scattering data to a bilayer model for the component groups ${ }^{16}$ as an alternative way to determine the position of both bioflavinoids in membranes. These structural and elasticity results provide insight into the effects of bioflavinoids on lipid membranes.

\section{MATERIALS AND METHODS}

Samples. Dioleoylphosphatidylcholine (DOPC) (lot 18:1228) and diphytanoyl-phosphatidylcholine (DPhyPC) (lots 4Me160-118, 4Me160-121) were purchased from Avanti Polar Lipids in the lyophilized form. Bioflavinoids, genistein and daidzein, were purchased from Sigma/Aldrich (St. Louis, MO); their chemical structures are shown in Figure 1. Lipid/

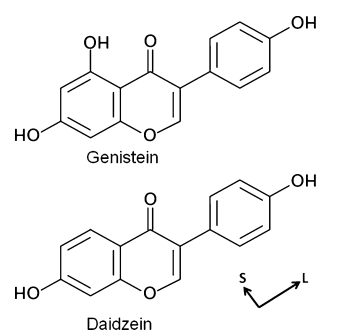

Figure 1. Chemical structures of the bioflavinoids, genistein and daidzein. The long (L) and short (S) axes of the bioflavinoids are shown.

bioflavinoid mixtures in the mole fractions $0.05,0.10,0.15$, and 0.20 were prepared by mixing with lipids in organic solvent. Bioflavinoids are insoluble in most common organic solvents at room temperature, including chloroform, methanol, acetone, hexafluoroisopropyl alcohol and trifluoroethanol, but soluble in hot methanol and DMSO.

DOPC/bioflavinoid mixtures (4 $\mathrm{mg}$, in duplicate) were dissolved in $200 \mu \mathrm{L}$ of HPLC hot methanol and plated onto 30 $\mathrm{mm} \times 15 \mathrm{~mm} \times 1 \mathrm{~mm}$ silicon wafers in an oven at $50{ }^{\circ} \mathrm{C}$ using the rock and roll technique. ${ }^{17}$ For DPhyPC/bioflavinoid mixtures, evaporation from chloroform in the fume hood was more successful than hot methanol at producing well-oriented samples. After drying overnight in the hood, samples were trimmed to a $5 \mathrm{~mm} \times 30 \mathrm{~mm}$ strip in the center of the wafer. Hydration of oriented samples from water vapor was then carried out in a thick-walled hydration chamber. ${ }^{18}$ Unoriented multilamellar vesicles (MLV) in excess water were prepared by weighing 1-2 mg of dry lipid mixture with $40 \mu \mathrm{L}$ of milli-Q water and thoroughly mixing in small nalgene vials, then thermally cycling three times with vortexing between -20 and $50{ }^{\circ} \mathrm{C}$ before loading into 1-mm-diameter glass capillaries (Charles Supper, Cambridge, MA). Thin layer chromatography using chloroform/methanol/7 $\mathrm{N} \mathrm{NH}_{4} \mathrm{OH}(46: 18: 3, \mathrm{v} / \mathrm{v})$ and a molybdic acid stain indicated negligible lysolipid before and $0.1-0.5 \%$ lysolipid after X-ray exposure, with genistein samples showing slightly more degradation than daidzein samples.

Volume Determination. Volumes in fully hydrated MLV were determined at $30 \pm 0.01{ }^{\circ} \mathrm{C}$ using an Anton-Paar USA DMA5000M (Ashland, VA) vibrating tube densimeter. The volume of the "unit cell", $V_{1}$, is defined to be the volume, $V_{\mathrm{L}}$, of one lipid plus the proportional bioflavinoid volume $V_{\mathrm{BF}} f /(1-$ $f)$, where the mole fraction, $f$, is the number of moles of bioflavinoid divided by the number of moles of lipid and bioflavinoid and $V_{\mathrm{BF}}$ is the volulme of one bioflavinoid. $V_{1}$ was calculated from

$$
V_{1}=\frac{\mathrm{MW}_{1}}{0.6022 \rho_{\mathrm{s}}}\left[1+\frac{m_{\mathrm{W}}}{m_{\mathrm{M}}}\left(1-\frac{\rho_{\mathrm{s}}}{\rho_{\mathrm{w}}}\right)\right]
$$

where $\rho_{\mathrm{S}}$ and $\rho_{\mathrm{W}}$ are the measured densities of the samples and pure water, respectively; $m_{\mathrm{W}}$ is the mass of water; and $m_{\mathrm{M}}$ is the sum of the masses of the lipid and the bioflavinoid in the mixture. In accordance with the definition of $V_{1}, \mathrm{MW}_{1}$ is the molecular weight of one lipid plus $f /(1-f)$ times the molecular weight of the bioflavinoid.

X-ray scattering. X-ray data from oriented fluid phase lipid mixtures at $30{ }^{\circ} \mathrm{C}$ were obtained on two trips to the Cornell High Energy Synchrotron Source (CHESS) using the G1 station managed by Dr. Arthur Woll. The wavelength was set with a $\mathrm{WB}_{4} / \mathrm{C}$ multilayer monochromator to $1.1803 \AA$ on trip 1 and to $1.1825 \AA$ on trip 2 , with a total beam intensity of $5 \times$ $10^{11}$ photons $/ \mathrm{s} / \mathrm{mm}^{2}$. Beam width was $0.26 \mathrm{~mm}$, and the beam height was $0.9-1.2 \mathrm{~mm}$. The samples were $\sim 10 \mu \mathrm{m}$ thick along the normal to the $\sim 2000$ bilayers. The angle of the flat samples was cycled uniformly from -3 to 7 and back to $-3^{\circ}$ relative to the beam once per second during the 30-60 s exposures. Data were collected using a Flicam CCD (Finger Lakes Instrumentation, Lima, NY) with a $1024 \times 1024$ pixel array with average pixel size $71 \mu \mathrm{m} /$ pixel. The sample-to-CCD distance was 371 or $354 \mathrm{~mm}$, calibrated using a silver behenate standard with $D$ spacing of $58.4 \AA$. The temperature was controlled with a Neslab Controller (Portsmouth, NH) and monitored using a Cole-Parmer thermistor (Vernon Hills, IL). To obtain fully hydrated $D$ spacings, MLV samples were X-rayed at CMU at 30 ${ }^{\circ} \mathrm{C}$ using a Rigaku RUH3R microfocus rotating anode (Woodlands, TX) equipped with Xenocs FOX2D (Sassenage, France) focusing collimation optics.

The analysis of diffuse data from oriented stacks of fluctuating fluid bilayers has been previously described ${ }^{18-21}$ and will only briefly be summarized here. The scattering intensity for a stack of oriented bilayers is the product: $I(\mathbf{q})=$ $S(\mathbf{q})\left|F\left(q_{z}\right)\right|^{2} / q_{z}$, where $\mathbf{q}=\left(q_{r}, q_{z}\right), S(\mathbf{q})$ is the structure interference factor, $F\left(q_{z}\right)$ is the bilayer form factor, and $q_{z}{ }^{-1}$ is the usual low-angle X-ray scattering (LAXS) approximation to the Lorentz factor for which all the sample remains in the beam for all relevant $q$. The first step of the analysis obtains the bilayer bending modulus, $K_{\mathrm{C}}$, and the compression modulus, $B$, by fitting to the $q_{r}$ dependence of the diffuse X-ray scattering. $\left|F\left(q_{z}\right)\right|^{2} / q_{z}$ is then determined by dividing $I(q)$ by the $S(\mathbf{q})$ derived from validated liquid crystal theory. A geometric undulation correction $^{22}$ is used to multipy the $q_{z}$ axis of $F\left(q_{z}\right)$ by a factor slightly larger than 1 ; for example, 1.02 .

Structural Analysis. The X-ray $\left|F\left(q_{z}\right)\right|$ data were fit using a recently devised modeling procedure abbreviated SDP for scattering density profile. ${ }^{16}$ This procedure satisfies the important principle of volume conservation, which requires that the volume probabilities of all the components sum to 1 at all $z$ along the bilayer normal. The SDP procedure guarantees an important relation between the area, $A_{1}$, and the zerothorder X-ray form factor, $F(0):^{23}$

$$
A_{1} F(0)=2\left(n_{1}-\rho_{\mathrm{W}} V_{1}\right)
$$

where $V_{1}$ is the measured volume of the lipid/bioflavinoid mixture in eq $1 ; n_{1}=n_{\mathrm{L}}+n_{\mathrm{BF}} f /(1-f)$ is the number of electrons; $n_{\mathrm{L}}$ is 434 for DOPC and 470 for DPhyPC; $n_{\mathrm{BF}}$ is 140 for genistein and 132 for daidzein; $\rho_{\mathrm{W}}=0.333 \mathrm{e} / \AA^{3}$ is the 
electron density of water at $30^{\circ} \mathrm{C}$; and $A_{1}$ is the area of the unit cell, similar to the above definition of $\mathrm{V}_{1}$. The original SDP application parsed the lipid molecule into components, called the SDP model, that were especially appropriate for simultaneous analysis of X-ray and neutron scattering data. ${ }^{16}$ For X-ray only data, in this paper, we have parsed the system differently, combining some of the features of the older $\mathrm{H}_{2}{ }^{24}$ and $\mathrm{HB}$ models. ${ }^{25}$ We represented the phosphatidylcholine part of the headgroup with a PC Gaussian, the carbonyl/glycerol part of the headgroup with a CG Gaussian, and the hydrocarbon chains with a combination of error functions and a Gaussian for the terminal methyls, as previously described. ${ }^{16}$ The bioflavinoid was represented by an additional Gaussian.

The fitting procedure allows the use of outside information to impose constraints on the model parameters. Informed by our volume measurements, we constrained the ratio of bioflavinoid volume to lipid volume, and the chain terminal methyl to the chain methylene volume ratio to 1.96 . Consistent with the MD simulations, we constrained the widths of the lipid headgroup peaks to $2.5 \AA$, the bioflavinoid width to $3.0 \AA$, the methyl trough width to $3.0 \AA$, and the width of the hydrocarbon interface to $2.4 \AA$. We also constrained $D_{\mathrm{H} 1}$, the difference in distance between the maximum in the electron density profile and the Gibbs dividing surface for the hydrocarbon region, to $4.95 \AA$ and $\Delta D_{\mathrm{H}}$, the difference in distance between the PC and CG Gaussians, to $4.6 \AA$. Otherwise, the locations of the components remained free to provide estimates for $D_{\mathrm{HH}}$ and the position of the bioflavinoid in the membrane.

MD Simulations. Release version c35b4 of the CHARMM $\operatorname{program}^{26}$ was used for all molecular dynamics simulations, model building, and either analysis of the simulation data or extraction of data for further analysis with other programs. Distributed CHARMM force field parameters were utilized for DOPC $^{27}$ and water; ${ }^{28}$ CHARMM-compatible parameters for genistein were obtained from Burendahl et al., ${ }^{29}$ and parameters for daidzein were derived by analogy from them. A forceswitched Lennard-Jones method was used for the van der Waals term of the potential, with a cutoff of $12 \AA$, and the switching function starting at $10 \AA$. Electrostatics were computed with the particle-mesh Ewald method using a $12 \AA$ real space cutoff, $\kappa=$ 0.32, a fifth-order spline interpolation for the complementary error function, and a grid density of $\sim 1$ Å. All MD simulations used the Verlet leapfrog integrator and the extended pressure system implemented in CHARMM.

Pure DOPC systems were either an extension to $105 \mathrm{~ns}$ of an NPT ensemble simulation from Klauda et al., ${ }^{27}$ or derived from that system and simulated via the NPAT ensemble (fixed area/ lipid) for $35 \mathrm{~ns}$. Starting with the NPT system ( $\langle$ area/lipid $\rangle=$ $\left.69.0 \AA^{2}\right)$, the number density $z$ profiles for each uniquely labeled atom of a molecule were first extracted from the simulation trajectories and then combined and formatted with a Fortran program for subsequent use as input to the SIMtoEXP program. ${ }^{30}$ The SIMtoEXP program compares X-ray structure factors calculated from scattering data with those calculated from the simulation number density profiles. In this case, the comparison indicated the area cross section (69 $\AA^{2} /$ lipid) was too small and that simulations at larger area/lipid were required to provide atomic level insight into the experimental results. Two additional DOPC simulations were generated in the NPAT ensemble, with fixed area/lipid values of 72.4 and $75 \AA^{2}$. The last $25 \mathrm{~ns}$ of these two simulations were used for data analysis, and the last $90 \mathrm{~ns}$ of the NPT simulation were included for the calculation of $K_{\mathrm{A}}$. For further model building, a library of individual DOPC conformations was derived from the latter part of the NPT simulation coordinate trajectory; random selection from this library was used to pack the mixtures described below.

Systems containing $20 \%$ bioflavinoids were constructed de novo by placing an oriented lipid or bioflavinoid at grid points in a hexagonal lattice via random selection of the molecule type. Using a spacing derived from initial area estimates from the scattering experiments, a $7 \times 7$ grid was used for each leaflet, placing 36 lipids and 9 bioflavinoids, with 4 unoccupied lattice points, giving a total of 72 lipids and 18 bioflavinoids. After successful packing, the systems were hydrated with TIP3P water, energy-minimized, adjusted to give a total water count of 2400 molecules (based on estimates of hydration for the experiments), and more extensively minimized. Replicate models for each bioflavinoid were built in this fashion, and three were chosen for NPT ensemble simulations. After heating from $203 \mathrm{~K}$, the Hoover thermostat at $303 \mathrm{~K}$ was employed for NPT simulations for a total run time of $45 \mathrm{~ns}$. Analogous to the case for DOPC, comparison of the experimental X-ray structure factor curves with those from the last $35 \mathrm{~ns}$ of the NPT simulations indicated that the simulation area cross sections (73-75 $\AA^{2} /$ lipid) were too small to match the experiments and that NPAT simulations at larger areas were required. Systems containing $14 \%$ daidzein were obtained by removing three bioflavinoids from each leaflet, energy-minimizing, adjusting the area cross section via $\mathrm{NP} \gamma \mathrm{T}$ simulations, and running for an additional 35 ns.

A genistein coordinate set from the 45 ns point of one of the NPT simulations was chosen as a starting point for further simulations at fixed areas of 80,83 , and $86 \AA^{2} /$ lipid. To expand the area cross section, short NP $\gamma \mathrm{T}$ simulations were used, with $\gamma=15 \mathrm{dyn} / \mathrm{cm} /$ leaflet sufficient to attain the target areas. Three matching daidzein conformations were created by modification of the genistein molecules (converting $-\mathrm{OH}$ to $-\mathrm{H}$ ). Six NPAT simulations were started, with each bioflavinoid simulated at each of the three areas given above. On the basis of a preliminary comparison to experimental X-ray structure factors, additional daidzein simulations were done at $A=78 \AA^{2} /$ lipid for mole fraction $f=0.20$ and at $A=79$ and 82 $\AA^{2} /$ lipid for $f=0.14$, as well as a genistein simulation with $A=$ $84.5 \AA^{2} /$ lipid at $f=0.20$. These 10 NPAT simulations were run for $35 \mathrm{~ns}$, and the final $25 \mathrm{~ns}$ of each was used for all data analysis.

\section{RESULTS}

Volumes. Table 1 shows our measured unit cell volume, $V_{1}(\exp )$, consisting of one lipid plus the fraction $f /(1-f)$ of a bioflavinoid. Addition of mole fraction $f=0.2$ bioflavinoids increased $V_{1}(\exp )$ compared with the molecular volumes of pure DOPC and DPhyPC measured previously. ${ }^{31-33}$ Table I also shows that our MD simulations for DOPC give values $V_{1}(\operatorname{sim})$ that agree very well with $V_{1}(\exp )$. If we assume that the volume of the lipid does not change upon addition of bioflavinoids, then we obtain the volumes $V_{\mathrm{BF}}$ (calcd) of genistein and daidzein listed in Table I. Another way to estimate the volumes $V_{\mathrm{BF}}$ employs a volumetric analysis of components in simulations. ${ }^{34}$ This gives the larger volumes $V_{\mathrm{BF}}(\operatorname{sim})$ for the bioflavinoids in DOPC shown in Table I and requires a corresponding condensation of the lipid volumes (not shown). 
Table 1. Tabulated Results ${ }^{a}$

\begin{tabular}{|c|c|c|c|c|c|c|}
\hline \multirow[b]{2}{*}{ lipid } & \multicolumn{6}{|c|}{ bioflavinoid } \\
\hline & none & Gen & Daid & none & Gen & Daid \\
\hline & \multicolumn{3}{|c|}{ DOPC } & \multicolumn{3}{|c|}{ DPhyPC } \\
\hline$f$ & 0 & 0.20 & 0.14 & 0 & 0.20 & 0.12 \\
\hline$V_{1}(\exp )$ & 1303 & 1385 & $1380^{b}$ & 1427 & 1518 & $1513^{b}$ \\
\hline$V_{1}(\operatorname{sim})$ & 1304 & 1384 & $1380^{b}$ & & & \\
\hline$V_{\mathrm{BF}}(\mathrm{calc})$ & & 328 & $308^{b}$ & & 363 & $343^{b}$ \\
\hline$V_{\mathrm{BF}}(\operatorname{sim})$ & & 437 & $352^{b}$ & & & \\
\hline$A_{1}(\exp )$ & 72 & 82 & 79 & 83 & 93 & 88 \\
\hline$A_{1}(\operatorname{sim})$ & 75 & 83 & 80 & & & \\
\hline$D_{\mathrm{HH}}(\exp )$ & 36.1 & 33.8 & 34.6 & 36.4 & 35.0 & 35.5 \\
\hline$D_{\mathrm{HH}}(\operatorname{sim})$ & 36 & 33.9 & 34.5 & & & \\
\hline$Z_{\mathrm{BF}}(\exp )$ & & 11.8 & 12.3 & & 13.0 & 13.4 \\
\hline$Z_{\mathrm{BF}}(\operatorname{sim})$ & & 11.8 & 12.3 & & & \\
\hline$K_{\mathrm{A}}(\operatorname{sim})$ & 250 & 160 & 190 & & & \\
\hline$K_{\mathrm{C}}(\exp )$ & 6.5 & 5.7 & 4.8 & 5.3 & 5.4 & 5.5 \\
\hline
\end{tabular}

${ }^{a}$ Units are appropriate powers of $\AA, \mathrm{mN} / \mathrm{m}$ for $K_{\mathrm{A}}, 10^{-20} \mathrm{~J}$ for $K_{\mathrm{C}} . f$ is mole fraction of bioflavinoid, Gen is genistein and Daid is daidzein, and subscript 1 signifies a unit cell consisting of 1 lipid and $f /(1-f)$ bioflavinoid. ${ }^{b}$ Results for $f=0.20$.

Structural X-ray Data. The lobes of diffuse scattering from oriented samples are shown numbered in Figure 2. The diffuse scattering for DOPC at $30{ }^{\circ} \mathrm{C}$ extends beyond $0.8 \AA^{-1}$ with three strong lobes $(1-3)$ and two lobes of weaker scattering

\section{DOPC}
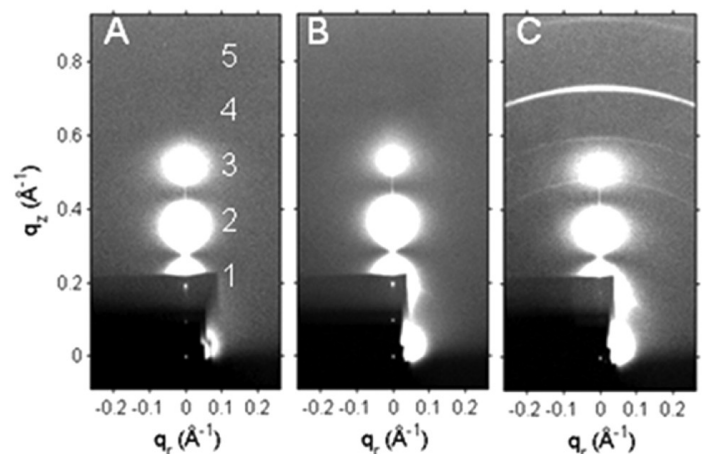

\section{DiphytanoyIPC}

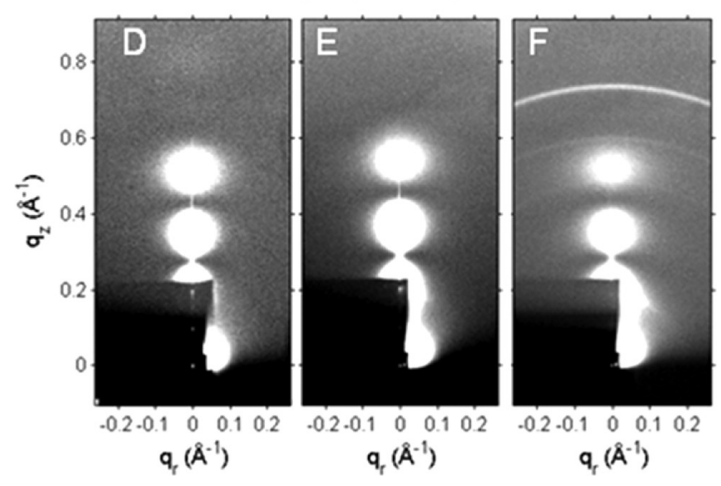

Figure 2. 2D CCD images of LAXS diffuse scattering; white is the highest intensity. (A) DOPC, (B) DOPC/20\% genistein, (C) DOPC/ $20 \%$ daidzein, (D) DPhyPC, (E) DPhyPC $/ 20 \%$ genistein, (F) DPhyPC $/ 20 \%$ daidzein. The dark shadows are caused by attenuators through which the beam and orders 1 and 2 can be seen. Lobes are numbered for DOPC. not visible in Figure 2A. The two weaker lobes (4 and 5) are barely visible for DPhyPC in Figure 2D. It is important to emphasize that accurate measurement of weak or zero intensity increases the spatial resolution of structural data. Our diffuse data contain information equivalent to $\sim 8$ orders of Bragg diffraction from rather dry samples and more than the usual 24 orders obtained in many investigations of more hydrated samples. There are also sharp peaks corresponding to orders $h$ $=1$ and 2 from which the lamellar $D$ spacing was obtained; these peaks are barely visible in Figure 2 because their intensities were reduced by a factor of $\sim 1700$ by a $0.1-\mathrm{mm}$ thick molybdenum attenuator.

As the relative humidity in the sample chamber was increased, the measured $D$ spacing increased toward the fully hydrated $D$ spacing determined from MLV samples in excess water in capillaries. These $D$ spacings did not change significantly with addition of bioflavinoids; their values are shown in Figure S1 of the Supporting Information. The diffuse X-ray data were collected within $5 \AA$ of the fully hydrated $D$ spacing. At the highest concentration $(f=0.20)$ of daidzein, Figure $2 \mathrm{C}$ and $\mathrm{F}$ shows several sharp reflections, presumably due to the presence of daidzein crystals. The measured $D$ spacing of the strongest sharp reflection was $8.4 \AA$, comparable to the length of a tilted daidzein molecule. These reflections were removed for the structural analysis, but this crystallization limited the concentration of bioflavinoid in this study. The ratio of the intensity of the sharp reflections to the intensity of the diffuse scattering was larger for our nominal $f=0.2$ daidzein samples than for our nominal $f=0.15$ daidzein samples. By extrapolating to zero intensity of the sharp rings (data not shown), we estimate that the solubility limit of daidzein in DOPC is $f=0.14$, and in DPhyPC, it is $f=0.12$ in these oriented samples. Crystal rings did not appear in any of the genistein samples.

Structural Results. Figure 3 compares the X-ray form factors obtained from MD simulations of $20 \mathrm{~mol} \%$ genistein in DOPC at three different unit cell areas, $A_{1}$. As $A_{1}$ increases and the thickness of the bilayer decreases, the simulated lobes of $\left|F\left(q_{z}\right)\right|$ move to larger $q_{z}$. Comparison with the experimental $\left|F\left(q_{z}\right)\right|$ obtained from the data in Figure $2 \mathrm{~B}$ indicates that the best simulated value of $A_{1}$ is closer to $83 \AA^{2}$ than to 80 or 86

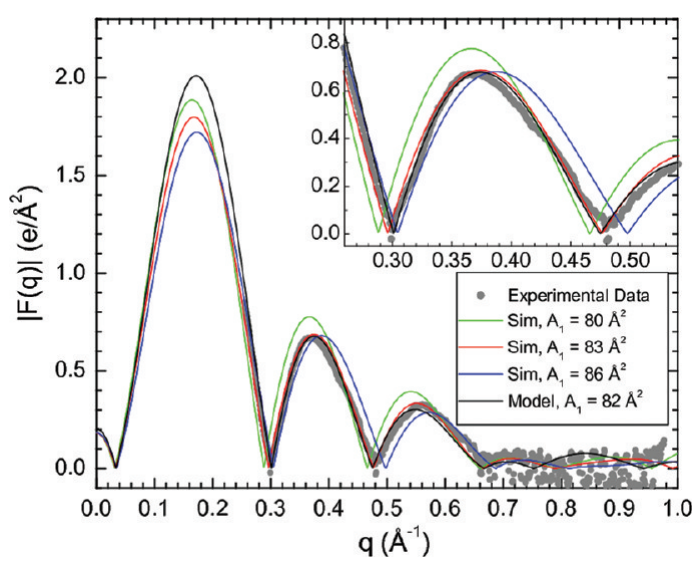

Figure 3. Experimental form factor $\left|F\left(q_{z}\right)\right|$ data for $20 \mathrm{~mol} \%$ genistein in DOPC (gray circles) compared with the form factors resulting from three MD NPAT simulations fixed to areas $A_{1}$ of 80 (green), 83 (red), and 86 (blue) $\AA^{2}$. Also shown is the model fit (black) that yielded $A_{1}=$ $82 \AA^{2}$. The inset shows more detail in the second lobe. 
$\AA^{2}$. Similar comparisons of simulations and experiment were performed for DOPC and 20\% (14\%) daidzein in DOPC to obtain the simulated values of $A_{1}$ shown in Table 1 . Figure 3 also shows $\left|F\left(q_{z}\right)\right|$ obtained from modeling. Of course, with a sufficiently complex model with many unconstrained parameters, it is possible to fit the experimental $\left|F\left(q_{z}\right)\right|$ quite well, but this yields component distributions in real space that are not realistic. It is therefore important that the model be constrained to be realistic, and the simulations are valuable for that, as we show next.

Figure 4 compares the real space electron density distributions of the DOPC lipid components and $20 \%$ genistein

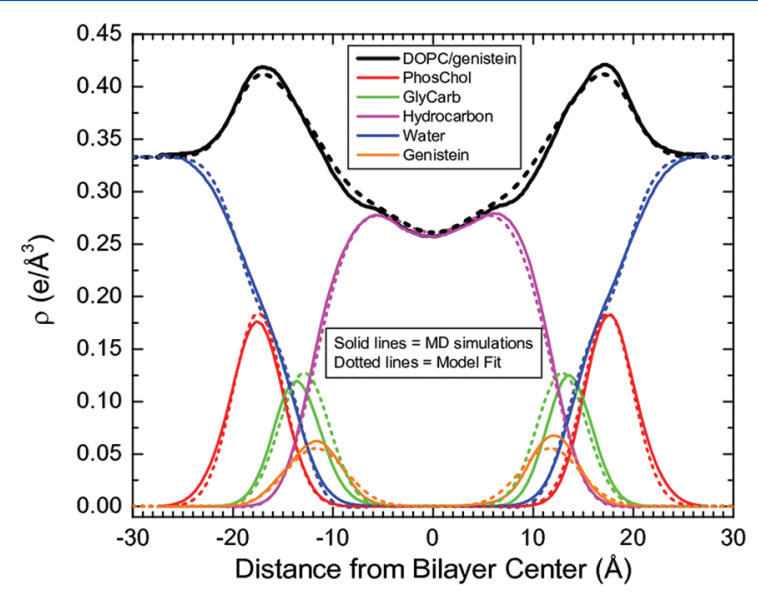

Figure 4. Electron density profiles for DOPC with $20 \%$ genistein resulting from $\mathrm{MD}$ simulation at $A_{1}=83 \AA^{2}$ (solid lines) and model fitting to experimental data (dashed lines). Component groups are identified by colors shown in the inset.

as obtained from the simulation at $A_{1}=83 \AA^{2}$ with those obtained from modeling of the experimental $\left|F\left(q_{z}\right)\right|$. Although the widths of the model distributions have been constrained, it is important to emphasize that the unconstrained positions $z_{\mathrm{BF}}$ of the modeled genistein and the headgroups are nearly the same as those of the simulations. This strongly supports the validity of the simulations. Table 1 shows the good agreement between the values of the simulated and experimental headhead spacing $D_{\mathrm{HH}}$ in the electron density profiles and the average position of both bioflavinoids.

Figure 5 shows the X-ray form factors $\left|F\left(q_{z}\right)\right|$ from oriented samples of DOPC and bioflavinoids, and Figure 6 shows the Xray form factors from oriented samples of DPhyPC and bioflavinoids that came from the CCD images shown in Figure 2. The solid curves in Figures 5 and 6 result from the model fits. The vertical dotted lines help compare the effects of the bioflavinoids on the positions of the zero between lobes 1 and 2. The data for $20 \%$ genistein in both DOPC and DPhyPC shifts to higher $q_{z}$ which is an indication of a thinning of the bilayers.

Figures 7 and 8 show electron density profiles obtained from modeling experimental data. The most robustly determined quantity is the head-to-head thickness, $D_{\mathrm{HH}}$, which is defined as the distance between the maxima in the total electron density profiles. The vertical dotted lines help to compare the effects of the bioflavinoids on $D_{\mathrm{HH}}$ of DOPC. Results for the highest concentrations of bioflavinoid are given in Table 1. Genistein decreased the thickness of DOPC somewhat more than daidzein, as shown in Figures 7 and 8 and quantified in

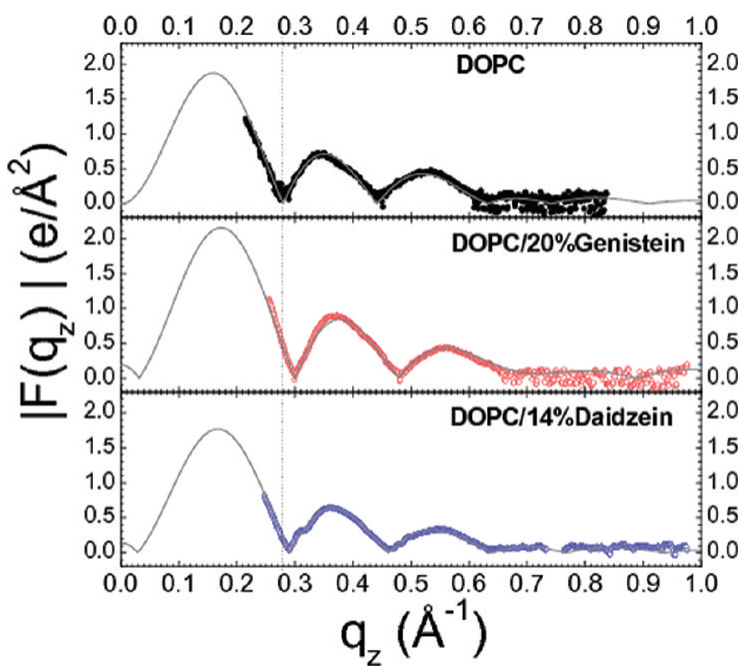

Figure 5. $\left|F\left(q_{z}\right)\right|$ data for DOPC (black), DOPC/20\% genistein (red) and DOPC $/ 14 \%$ daidzein (blue).

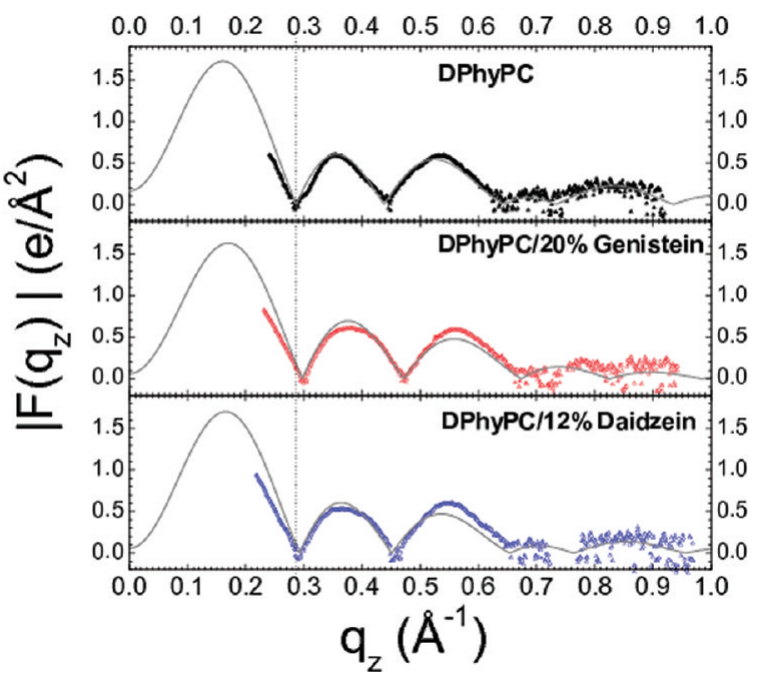

Figure 6. $\left|F\left(q_{z}\right)\right|$ data for DPhyPC (black), DPhyPC/20\% genistein (red) and DPhyPC/12\% daidzein (blue).

Table 1. With increasing concentration, genistein moved slightly toward the bilayer center, and daidzein moved slightly outward from the bilayer center. Similar but smaller thinnings were observed when these bioflavinoids were added to DPhyPC, as shown in Figures S2 and S3 in the Supporting Information and quantified in Table 1. Figures S2 and S3 also note slight movements in the bioflavinoids in DPhyPC with increasing concentration.

Elasticity. Results for the bending modulus, $K_{C}$, are shown in Figure 9. The $K_{\mathrm{C}}$ values were averaged from data at different $D$ spacings from the same sample as well as from different samples. For any one sample, $K_{C}$ did not vary systematically with $D$ spacing, consistent with its being a property of single bilayers. As shown in Figure 9, the effect of either bioflavinoid on $K_{\mathrm{C}}$ was relatively small, especially in the case of genistein.

The values of the area compressibility modulus, $K_{\mathrm{A}}=$ $2 A_{1}\left(\partial \gamma / \partial A_{1}\right)$, given in Table 1 were obtained from the simulated results for the leaflet surface tension, $\gamma$, in Figure 10. $K_{\mathrm{A}}$ was clearly smaller with either bioflavinoid than for the DOPC 


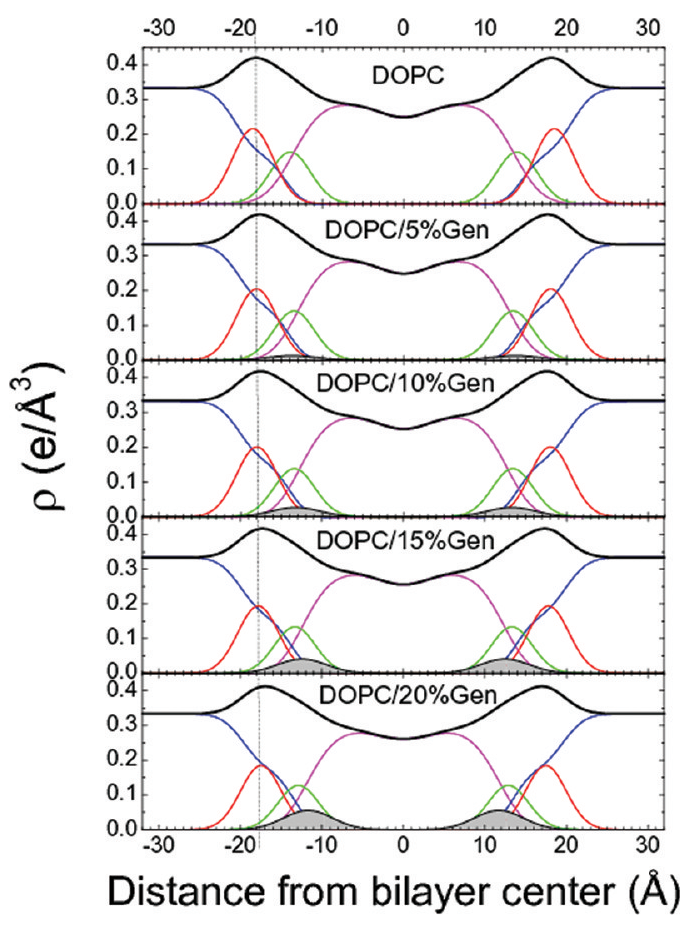

Figure 7. Electron density profiles of DOPC with increasing concentration of genistein obtained using the SDP procedure. Component groups are phosphate (red), carbonyl-glycerol (green), methylenes and terminal methyl group (magenta), water (blue), bioflavinoid (filled gray), and total (black).

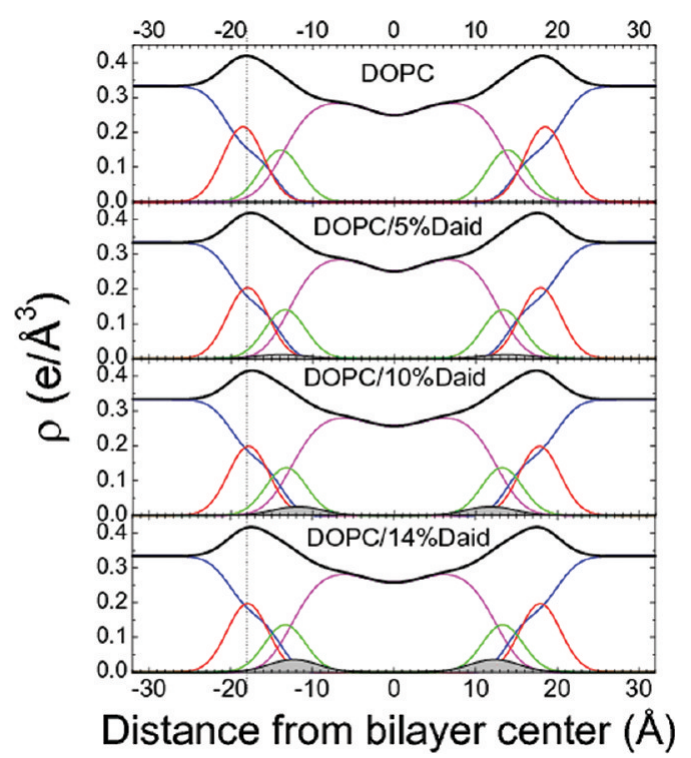

Figure 8. Electron density profiles of DOPC with increasing concentration of daidzein obtained using the SDP procedure. Line colors as for Figure 7.

control. As determined from the slopes in Figure 10, $K_{\mathrm{A}}$ for $f=0.14$ daidzein $(190 \mathrm{mN} / \mathrm{m})$ was closer to the control value $(240 \mathrm{mN} / \mathrm{m})$ than the $K_{\mathrm{A}}$ for $f=0.20$ genistein $(160 \mathrm{mN} / \mathrm{m})$.

Figure 11 shows two snapshots from the NPAT simulations. Electron density profiles resulting from the simulations at three fixed areas are shown in Figure S4 in the Supporting Information.

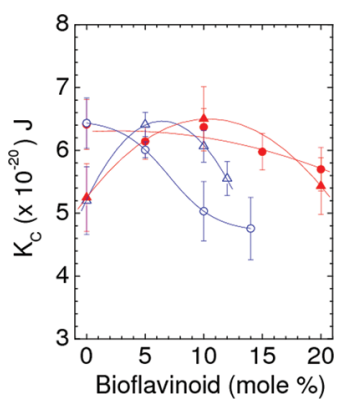

Figure 9. Bending modulus, $K_{C}$, vs concentration of genistein (solid symbols) and daidzein (open symbols) in DOPC (circles) and DPhyPC (triangles).

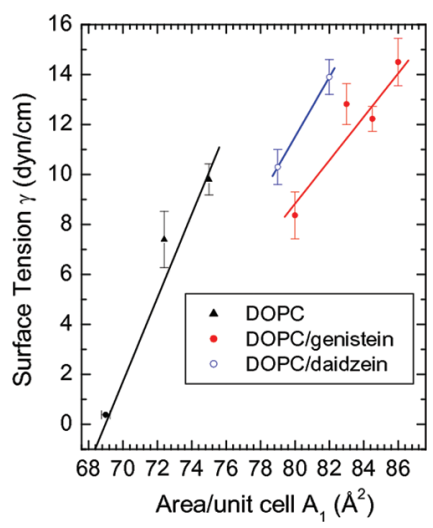

Figure 10. Surface tension, $\gamma$, vs $A_{1}$ for DOPC with and without 20 mol \% genistein and $14 \mathrm{~mol} \%$ daidzein from NPAT simulations except the $\gamma=0$ point for pure DOPC, which was an NPT simulation.

\section{DISCUSSION}

This study emphasizes the synergy between experiments and $\mathrm{MD}$ simulations. Although the X-ray method provides firm structural data to the highest resolution possible in fully fluid lipid bilayer systems, the extraction of meaningful structural results becomes difficult upon the incorporation of additives that require more modeling parameters. On the other hand, simulations involve uncertainties in the force fields of the additives, and there are concerns that simulation times may not be long enough for additives to equilibrate to their experimental locations.

The main test of the simulations in this paper is that the Fourier transforms of the real space electron density profiles agree with the X-ray $\left|F\left(q_{z}\right)\right|$ data. Best agreement was obtained by adjusting the unit cell areas $A_{1}$ in NPAT simulations, and this then provides estimates of $A_{1}$, the head-head thickness $D_{\mathrm{HH}}$, and the positions $z_{\mathrm{BF}}$ of the bioflavinoids within the bilayer. The thickness, $D_{\mathrm{HH}}$, was obtained from the experimental data with a minimal amount of modeling. The good agreement of the simulations for $D_{\mathrm{HH}}$ is an important test of our procedure for obtaining $A_{1}$ from the simulations. Importantly, for direct modeling of the experimental data, the simulations provide the widths of the distributions of the bioflavinoids and the lipid component groups; this means that the modeling does not require so many totally free parameters. Without imposing simulation results for $A_{1}$ or $\mathrm{z}_{\mathrm{BP}}$, the modeling of the experimental $\left|F\left(q_{z}\right)\right|$ data gives good agreement with the simulations for those quantities.

We find both genistein and daidzein located closer to the headgroup than to the center of the bilayer, as qualitatively 


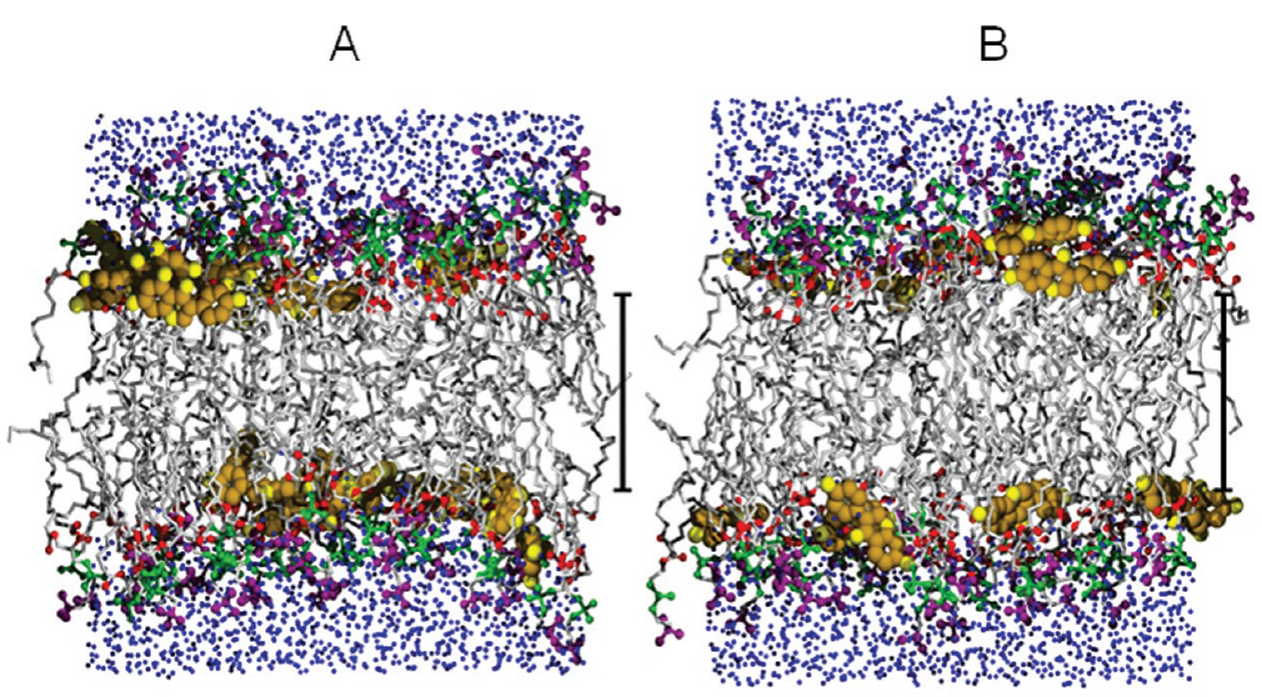

Figure 11. Snapshots of the MD simulations for (A) DOPC $/ 20 \mathrm{~mol} \%$ genistein at $A_{1}=83 \AA^{2}$ and for (B) DOPC $/ 14$ mol \% daidzein at $A_{1}=79 \AA^{2}$; each snapshot is 1 out of 25000 coordinate sets that comprise the ensemble; (A) collected at $30 \mathrm{~ns}$ ( $5 \mathrm{~ns}$ before the end of the simulation) and (B) 35 ns. Color coding: hydrocarbon chains, gray; bioflavinoids, gold and with yellow oxygen atoms; lipid carbonyl oxygens, red; phosphate group, green; choline group, purple; and water, blue. The scale bar marks 20 Å.

shown in Figure 11. For quantitative evaluation of differences, we use our values of $D_{\mathrm{HH}} / 2$ as a measure for the surface of the bilayer. Then the depth of insertion $D_{\mathrm{HH}} / 2-z_{\mathrm{BF}}$ from Table 1 is only $\sim 0.1 \AA$ greater for genistein than for daidzein in DOPC, although it is $\sim 1.1 \AA$ greater in DPhyPC. These small differences in depth of penetration are consistent with genistein's being more hydrophobic than daidzein. At first, this seems contrary to genistein's having an additional $\mathrm{OH}$ group. However, that extra $\mathrm{OH}$ group can participate in intramolecular hydrogen bonding with the adjacent carbonyl (Figure 1), thereby decreasing the hydrophilicity of the carbonyl on genistein relative to that of daidzein.

Our simulation indicates this intramolecular hydrogen bond is formed $95 \%$ of the time. Intramolecular hydrogen bonding in genistein is supported by ${ }^{1} \mathrm{H}$ NMR line width experiments of these bioflavinoids in SDS micelles, ${ }^{35}$ and the relative hydrophobicities are supported by the partitioning of genistein into octanol $(\log P=3.04)$ being greater than for daidzein $(\log \mathrm{P}=2.51) .{ }^{36}$ Consistently, the $\mathrm{C}=\mathrm{O}$ was associated with a water hydrogen $50 \%$ of the time for daidzein and only $25 \%$ of the time for genistein. Histograms of the bioflavinoid center of mass $|z|$ coordinate (Figure S5 in the Supporting Information) also suggest genistein is slightly more hydrophobic; the distribution outliers, shown by enlarging the baseline in the inset, are inward for genistein and outward for daidzein.

It is also especially encouraging that the difference between the volumes $V_{\mathrm{BF}}$ (calcd) of the two bioflavinoids shown in Table 1 are the same in DOPC and DPhyPC and that these differences are reasonable, given the replacement of $\mathrm{COH}$ in genistein by $\mathrm{CH}$ in daidzein. However, it seems unlikely that the volume of either bioflavinoid is so much different, as given in Table 1, in DPhyPC compared to DOPC. More likely is that either bioflavinoid alters the volume of different lipids by different amounts. For example, if genistein condensed DOPC to $1294 \AA^{3}$ and did not condense DPhyPC at all, then its volume would be $363 \AA^{3}$ in both lipids. We attempted to obtain some perspective on this from our simulations. The fact that the simulations agreed so well with the experimental "unit cell" volumes in Table 1 is another important test of the simulations. This success suggested trying to obtain the bioflavinoid volume using a well established component volume method. However, as noted in the original paper, ${ }^{34}$ high accuracy cannot be expected from this method when there are closely overlapping distributions; indeed, the results for $V_{\mathrm{BF}}(\operatorname{sim})$ listed in Table 1 yield the unlikely result that genistein is much larger than daidzein. Nevertheless, these results are consistent with the bioflavinoids' condensing rather than expanding DOPC. We propose that the values for $V_{\mathrm{BF}}(\mathrm{calcd})$ shown in Table 1 for DPhyPC are likely to be good estimates when bioflavinoids are added to most lipid bilayers.

Experimental validation of the simulation allows us to extract additional structural information that cannot be obtained from our experimental data. The narrow distributions of the bioflavinoids along the $z$ direction (Figure S5 of the Supporting Information) are consistent with their long axes being preferentially parallel to the bilayer surface (see Figure 1 for definition of axes). This is further confirmed in the simulation by the two hydroxyl oxygens at opposite ends of the bioflavinoids having nearly equal average distances from the center of the bilayer and by directly obtaining the angle $\beta$ of the long axes relative to the bilayer normal, shown in Figure 12B.

Hydrogen bonding of these two hydroxyls to water or phosphate in the interfacial region is the likely interaction responsible for this orientation, as well as for maintaining the location of the bioflavinoids close to the bilayer surface. The simulation also provides the angle, $\alpha$, of the short axes of the bioflavinoids around their long axes. The distribution of $\alpha$, shown in Figure 12A, is peaked about $\alpha=0$, which we define to be the value of $\alpha$ at which the plane of the rings is perpendicular to the bilayer surface and the $\mathrm{C}=\mathrm{O}$ points toward the proximal bilayer surface.

Study of the elastic properties also benefits from the synergy of experiment and simulation in that our experiments provide the bending modulus, $K_{\mathrm{C}}$, and our simulations provide the area compressibility modulus, $K_{\mathrm{A}}$. These two moduli are often related by

$$
K_{\mathrm{C}}=K_{\mathrm{A}}\left(2 D_{\mathrm{C}}\right)^{2} / N
$$



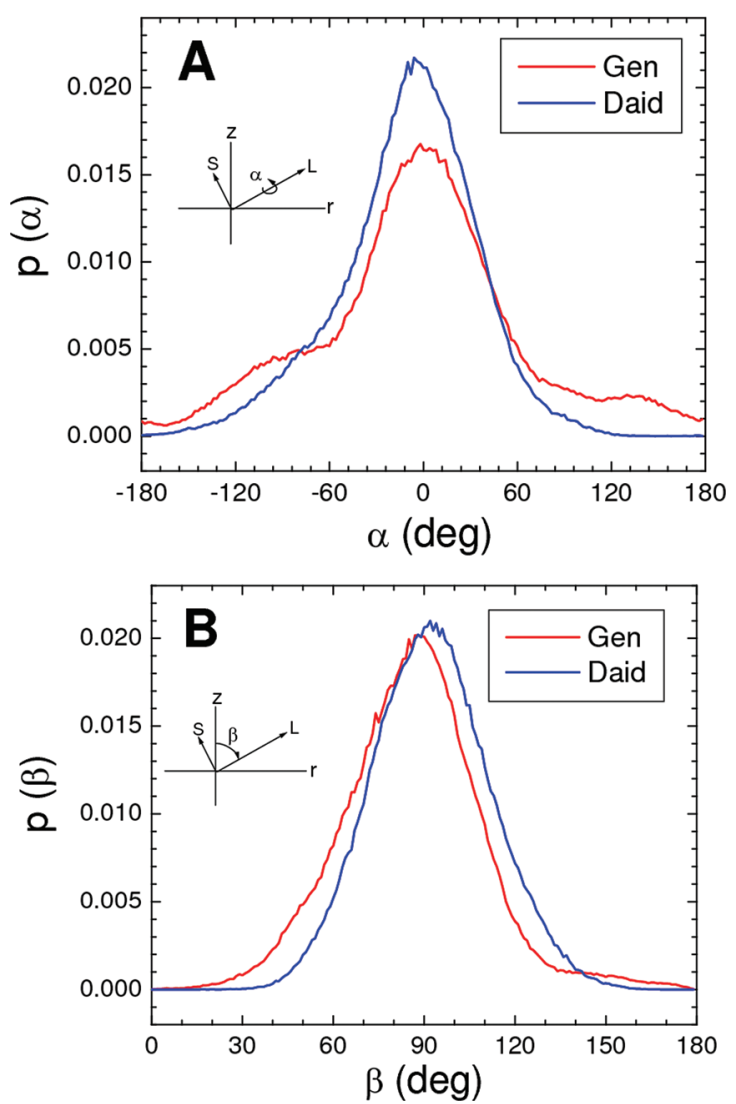

Figure 12. Probability histograms of bioflavinoids in DOPC membranes from the MD simulations for (A) the angle $\alpha$ of the short axis about the long axis and (B) the angle $\beta$ of the long axis with respect to the bilayer normal. Colors are $20 \mathrm{~mol} \%$ genistein, red; and $14 \mathrm{~mol} \%$ daidzein, blue. The insets show $\alpha$ and $\beta$ with respect to the long (L) and short (S) axes of the bioflavinoids, depicted in Figure 1.

where $2 D_{\mathrm{C}}$ is the hydrocarbon thickness. The value $N=24$ from the polymer brush theory fits experimental data for pure lipid bilayers quite well, ${ }^{37}$ although the relation breaks down as cholesterol is added. ${ }^{38}$ We observed that, within uncertainty, both bioflavinoids decreased $K_{\mathrm{A}}$ relative to the control DOPC by about the same $40 \%$ when either bioflavinoid concentration was $20 \%$ and proportionately less with $14 \%$ daidzein. In contrast, $K_{\mathrm{C}}$ remained nearly constant for genistein and decreased only about $20 \%$ for daidzein, so satisfying eq 3 would require $D_{\mathrm{C}}$ to increase considerably. However, if we define $D_{\mathrm{C}}$ to include only the hydrocarbons of the lipid, then our simulated $D_{\mathrm{C}}$ decreases from $13.0 \AA$ for DOPC alone to $11.8 \AA$ with $20 \%$ genistein and to $12.6 \AA$ with $14 \%$ daidzein. Even if we define $D_{\mathrm{C}}$ to include the largely hydrophobic bioflavinoids, $D_{\mathrm{C}}$ remains at $13.0 \AA$ with $20 \%$ genistein and increases to $13.6 \AA$ with $20 \%$ daidzein, so we conclude that eq 3 breaks down. Interestingly, eq 3 breaks down in the opposite way as for addition of cholesterol. ${ }^{38}$ For DOPC, cholesterol increases $K_{\mathrm{A}}$ and $2 D_{\mathrm{C}}$ while keeping $K_{\mathrm{C}}$ the same, so the righthand side of eq 3 becomes larger than the left-hand side for cholesterol, rather than smaller, as we find for bioflavinoids. This contrasting elastic behavior appears to be correlated with the structural difference that the long axis of cholesterol is preferentially oriented parallel to the bilayer normal, whereas the long axis of the bioflavinoids is preferentially oriented parallel to the bilayer surface.
The largest difference that we observe between the effects of genistein and daidzein on the DOPC bilayer, both in modeling of experimental data and in our simulations, is in the area of the unit cell, $A_{1}$. The average increase of $A_{1}(\exp )$ and $A_{1}(\operatorname{sim})$ for $20 \mathrm{~mol} \%$ genistein is $\sim 12 \%$ increase and $\sim 8 \%$ for daidzein at its solubility limit of $14 \mathrm{~mol} \%$. In DPhyPC, the increase of $A_{1}(\exp )$ is also an $\sim 12 \%$ increase for $20 \mathrm{~mol} \%$ genistein and $\sim 8 \%$ for $12 \%$ daidzein at its solubility limit. Therefore, the area increase is proportional to the concentration of either bioflavinoid in either lipid. These increases may be compared with the $6 \%$ increase reported for $20 \mathrm{~mol} \%$ of another bioflavinoid, curcumin, added to DOPC. ${ }^{39}$

One motivation for this work was to test the attractive hypothesis that the increased gA ion-channel lifetimes induced by bioflavinoids could be due to genistein's reducing the energy of hydrophobic mismatch of the gA channel to the lipid bilayer by reducing the appropriate elastic moduli, thereby making the bilayer softer and more easily deformable. By consideration of the details of deformations, the appropriate elasticity moduli have been proposed to be $K_{\mathrm{C}}{ }^{40}$ and both $K_{\mathrm{C}}$ and $K_{\mathrm{A} \cdot}{ }^{41} \mathrm{~A}$ simpler expression has been given ${ }^{14,42-44}$ as

$$
\Delta G_{\text {bilayer }}=H_{\mathrm{B}}\left(L-d_{0}\right)^{2}
$$

where $L$ is hydrophobic channel length, $d_{0}$ is the bilayer hydrocarbon thickness, and $H_{\mathrm{B}}$ is a phenomenological spring constant to mimic the effects of both $K_{\mathrm{C}}$ and $K_{\mathrm{A}}$. Decreasing $K_{\mathrm{C}}, K_{\mathrm{A}}$ or both would decrease $H_{\mathrm{B}}$ and $\Delta G_{\text {bilayer }}$, which, according to the hypothesis, would then increase channel lifetimes, as was observed. The hypothesis is therefore broadly consistent, for either bioflavinoid, with our results for $K_{\mathrm{A}}$ and $K_{\mathrm{C}}$. One might also consider that bioflavinoids could induce monolayer intrinsic curvature, but Lundbaek et al. reported that changes in monolayer intrinsic curvature do not play a major role. $^{12}$

Another important hypothesis that explains how bioflavinoids could increase gA channel lifetimes follows from our result for $D_{\mathrm{HH}}$ in Table 1 that bioflavinoids thin lipid bilayers. The hydrocarbon thickness, $d_{0}$, has been given as $2 D_{\mathrm{C}}=27.1 \AA$ for DOPC $^{22}$ and $27.2 \AA$ for DPhyPC, ${ }^{45}$ and the hydrophobic channel length, $L$, of wild-type gA has been given as $22 \AA^{13}$, so bioflavinoids would reduce the hydrophobic mismatch free energy in eq 4 by reducing $L-d_{0}$. Of course, the average thinning will be smaller for the lower concentrations used in gA experiments, ${ }^{14}$ but local thinning near a bioflavinoid could be comparable to the results in Table 1 . Such thin local regions would then provide an effectively attractive interaction between the bioflavinoids and gA dimers. Once formed, gA dimers with neighboring bioflavinoids would be relatively more stable with respect to breaking into gA monomers, and this would increase channel lifetimes.

However, neither of the preceding hypotheses is consistent with the reported experimental result that genistein is twice as effective as daidzein for increasing gA channel lifetimes. ${ }^{14}$ Regarding the first hypothesis, our results are that the decrease in $K_{\mathrm{A}}$ is nearly the same for both bioflavinoids up to the solubility limit of daidzein and that there is an even larger decrease in $K_{\mathrm{C}}$ for daidzein on a per mole basis, so daidzein would be expected to be even more effective than genistein for increasing channel lifetimes. Regarding the second hypothesis, we find that thinning is nearly the same for daidzein and genistein on a per mole basis. Because the concentration of daidzein in the gA lifetime experiments was about 4 times 
smaller than the limiting mole fraction $f=0.14$ (calculation given in the Supporting Information), the solubility limit does not account for the difference in lifetime results. Instead, the reason that genistein has a greater effect than daidzein on channel lifetimes is much simpler. The bioflavinoid concentrations reported in the gA channel lifetime experiments were based on the total amount of gA added to the system consisting of a black lipid membrane with its associated decane annulus and approximately 1000 times as much water by volume. Because genistein is more hydrophobic than daidzein, more of it partitioned into the bilayer when the overall reported concentrations were equal. From reported values of the partition coefficient, the $\log P$ of 3.04 for genistein and 2.51 for daidzein ${ }^{36}$ would give three times as much genistein as daidzein in the bilayer for equal aqueous concentrations. However, the finite ratio of aqueous to bilayer volume in the gA experiments ${ }^{14}$ alters the relative concentrations in the bilayer from a factor of 3 to a factor of 2 when equal overall amounts of bioflavinoid are added (see the Supporting Information for the calculation). This alone accounts for the differences in the effect of the bioflavinoids genistein and daidzein on gA channel lifetimes.

\section{SUMMARY AND CONCLUSIONS}

This work combines X-ray diffuse scattering, volume measurements, and $\mathrm{MD}$ simulations to determine structural and elastic properties of DOPC and DPhyPC bilayers with the bioflavinoids genistein and daidzein. The unit cell volume $V_{1}$ obtained by direct measurements of lipid/bioflavinoid mixtures is in excellent agreement with $V_{1}$ obtained from the MD simulations. There is good agreement between the electron density profiles and bioflavinoid positions obtained by MD simulations and model fits to the experimental form factor data. Both genistein and daidzein are located at the surface of the hydrocarbon region near the glycerol/carbonyl with the long axis parallel to the bilayer and the $\mathrm{C}=\mathrm{O}$ preferentially pointing toward the bilayer surface. Genistein thins DOPC by $\sim 2.3 \AA$ at $20 \mathrm{~mol} \%$, and daidzein thins DOPC by $\sim 1.5 \AA$ at $14 \mathrm{~mol} \%$.

Consistent with a theory for the effect of bioflavinoids on gramicidin lifetimes, both bioflavinoids generally softened bilayers. However, since the bending modulus, $K_{\mathrm{C}}$, decreased more with daidzein than with genistein and $K_{\mathrm{A}}$ decreased the same for both on a per mole basis, softening of the membrane is not the reason that genistein increases gA channel lifetimes twice as much as daidzein. Similarly, our result that both bioflavinoids thin the bilayer is consistent with the other hypothesis that bioflavinoids alleviate the hydrophobic free energy penalty for forming gA channels, but our result that the thinning is the same on a per mole basis is inconsistent with the larger reported effect of genistein. That factor of 2 is quantitatively explained by the relative water/hydrocarbon partition coefficients. ${ }^{36}$ Our simulation results and our experimental solubility limit results for daidzein are consistent with the partition coefficient result that genistein is more hydrophobic than daidzein.

\section{ASSOCIATED CONTENT}

\section{S Supporting Information}

Five figures that have been described in the main paper and calculations for the concentration of bioflavinoids in lipid membranes. This material is available free of charge via the Internet at http://pubs.acs.org.

\section{AUTHOR INFORMATION}

\section{Corresponding Author}

*Phone: 412-268-3174. Fax: 412-681-0648. E-mail: stn@cmu. edu.

\section{Present Addresses}

${ }^{\S}$ Department of Physics and Astronomy, Rice University, 6100 Main St., Houston, TX 77005

${ }^{\perp}$ Capital IQ, 55 Water St., New York, NY 10041

\section{Notes}

The authors declare no competing financial interest.

\section{ACKNOWLEDGMENTS}

This research was supported in part by Grant No. GM 44976 from NIGMS/NIH (S.T.-N., J.N.), the Howard Hughes Medical Institute (M.R., Y.Z.), and the Charles E. Kaufman Foundation (S.T.-N.). It was supported in part (R.V., R.P.) by the Intramural Research Program of the NIH, National Heart, Lung and Blood Institute and utilized the high-performance computational capabilities at the National Institutes of Health, Bethesda, MD (NHLBI LoBoS cluster). X-ray scattering data were taken at the Cornell High Energy Synchrotron Source (CHESS), which is supported by the National Science Foundation and the National Institutes of Health/National Institute of General Medical Sciences under National Science Foundation Award DMR-0225180. We especially thank Dr. Arthur Woll for obtaining our beam and for general support during our data collection at the G1 station. We thank Dr. Jianjun Pan for help with sample preparation and X-ray data collection at CHESS, Prof. Olaf Andersen for helpful discussions, and especially Dr. Gilman Toombes for very insightful criticism of a first draft.

\section{REFERENCES}

(1) Dixon, R. A.; Ferreira, D. Phytochemistry 2002, 60, 205-211.

(2) Birt, D. F.; Hendrich, S.; Wang, W. Q. Pharmacol. Ther. 2001, 90, 157-177.

(3) Derand, R.; Bulteau-Pignoux, L.; Becq, F. J. Biol. Chem. 2002, 277, 35999-36004.

(4) Akiyama, T.; Ishida, J.; Nakagawa, S.; Ogawara, H.; Watanabe, S.; Itoh, N.; Shibuya, M.; Fukami, Y. J. Biol. Chem. 1987, 262, 5592-5595.

(5) Bishop, J. M. Annu. Rev. Biochem. 1983, 52, 301-354.

(6) Coward, L.; Barnes, N. C.; Setchell, K. D. R.; Barnes, S. J. Agric. Food Chem. 1993, 41, 1961-1967.

(7) Kuiper, G. G. J. M.; Carlsson, B.; Grandien, K.; Enmark, E.; Haggblad, J.; Nilsson, S.; Gustafsson, J. A. Endocrinology 1997, 138, $863-870$.

(8) Kuiper, G. G. J. M.; Lemmen, J. G.; Carlsson, B.; Corton, J. C.; Safe, S. H.; van der Saag, P. T.; van der Burg, P.; Gustafsson, J. A. Endocrinology 1998, 139, 4252-4263.

(9) Rickard, D. J.; Subramaniam, M.; Spelsberg, T. C. J. Cell. Biochem. 1999, 123-132.

(10) Fanti, P.; Monier-Faugere, M. C.; Geng, Z.; Schmidt, J.; Morris, P. E.; Cohen, D.; Malluche, H. H. Osteoporosis Int. 1998, 8, 274-281.

(11) Palacios, V. G.; Robinson, L. J.; Borysenko, C. W.; Lehmann, T.; Kalla, S. E.; Blair, H. C. J. Biol. Chem. 2005, 280, 13720-13727.

(12) Lundbaek, J. A.; Birn, P.; Tape, S. E.; Toombes, G. E. S.; Sogaard, R.; Koeppe, R. E.; Gruner, S. M.; Hansen, A. J.; Andersen, O. S. Mol. Pharmacol. 2005, 68, 680-689.

(13) Hwang, T. C.; Koeppe, R. E.; Andersen, O. S. Biochemistry 2003 42, 13646-13658.

(14) Lundbaek, J. A.; Koeppe, R. E.; Andersen, O. S. Proc. Natl. Acad. Sci. U.S.A. 2010, 107, 15427-15430.

(15) Lehtonen, J. Y. A.; Adlercreutz, H.; Kinnunen, P. K. J. Biochim. Biophys. Acta, Biomembr. 1996, 1285, 91-100. 
(16) Kučerka, N.; Nagle, J. F.; Sachs, J. N.; Feller, S. E.; Pencer, J.;

Jackson, A.; Katsaras, J. Biophys. J. 2008, 95, 2356-2367.

(17) Tristram-Nagle, S. A. Methods Mol. Biol. 2007, 400, 63-75.

(18) Kučerka, N.; Liu, Y. F.; Chu, N. J.; Petrache, H. I.; TristramNagle, S.; Nagle, J. F. Biophys. J. 2005, 88, 2626-2637.

(19) Lyatskaya, Y.; Liu, Y. F.; Tristram-Nagle, S.; Katsaras, J.; Nagle, J. F. Phys. Rev. E 2001, 63, 0119071-0119079.

(20) Liu, Y. F.; Nagle, J. F. Phys. Rev. E 2004, 69, 040901040904(R).

(21) Kučerka, N.; Tristram-Nagle, S.; Nagle, J. F. Biophys. J. 2006, 90, L83-L85.

(22) Nagle, J. F.; Tristram-Nagle, S. Biochim. Biophys. Acta, Rev. Biomembr. 2000, 1469, 159-195.

(23) Nagle, J. F.; Wiener, M. C. Biophys. J. 1989, 55, 309-313.

(24) Klauda, J. B.; Kučerka, N.; Brooks, B. R.; Pastor, R. W.; Nagle, J. F. Biophys. J. 2006, 90, 2796-2807.

(25) Wiener, M. C.; Suter, R. M.; Nagle, J. F. Biophys. J. 1989, 55, $315-325$.

(26) Brooks, B. R.; Brooks, C.L. 3rd; Mackerell, A. D. Jr.; Nilsson, L.; Petrella, R. J.; Roux, B.; Won, Y.; Archontis, G.; Bartels, C.; Boresch, S.; Caflisch, A.; Caves, L.; Cui, Q.; Dinner, A. R.; Feig, M.; Fischer, S.; Gao, J.; Hodoscek, M.; Im, W.; Kuczera, K.; Lazaridis, T.; Ma, J.; Ovchinnikov, V.; Paci, E.; Pastor, R. W.; Post, C. B.; Pu, J. Z.; Schaefer, M.; Tidor, B.; Venable, R. M.; Woodcock, H. L.; Wu, X.; Yang, W.; York, D. M.; Karplus, M. J. Comput. Chem. 2009, 30, 15451614.

(27) Klauda, J. B.; Venable, R. M.; Freites, J. A.; O'Connor, J. W.; Tobias, D. J.; Mondragon-Ramirez, C.; Vorobyov, I.; MacKerell, A. D. Jr.; Pastor, R. W. J. Phys. Chem. B 2010, 114, 7830-7843.

(28) Jorgensen, W. L.; Chandrasekhar, J.; Madura, J. D.; Impey, R. W.; Klein, M. L. J. Chem. Phys. 1983, 79, 926-935.

(29) Burendahl, S.; Danciulescu, C.; Nilsson, L. Proteins 2009, 77, $842-856$.

(30) Kučerka, N.; Katsaras, J.; Nagle, J. F. J. Membr. Biol. 2010, 235, 43-50.

(31) Greenwood, A. I.; Tristram-Nagle, S.; Nagle, J. F. Chem. Phys. Lipids 2006, 143, 1-10.

(32) Tristram-Nagle, S.; Petrache, H. I.; Nagle, J. F. Biophys. J. 1998, $75,917-925$.

(33) Tristram-Nagle, S.; Kim, D. J.; Akhunzada, N.; Kucerka, N.; Mathai, J. C.; Katsaras, J.; Zeidel, M.; Nagle, J. F. Chem. Phys. Lipids 2010, 163, 630-637.

(34) Petrache, H. I.; Feller, S. E.; Nagle, J. F. Biophys. J. 1997, 72, 2237-2242.

(35) Whaley, W. L.; Rummel, J. D.; Kastrapeli, N. Langmuir 2006, 22, $7175-7184$.

(36) Rothwell, J. A.; Day, A. J.; Morgan, M. R. A. J. Agric. Food Chem. 2005, 53, 4355-4360.

(37) Rawicz, W.; Olbrich, K. C.; McIntosh, T.; Needham, D.; Evans,

E. Biophys. J. 2000, 79, 328-339.

(38) Pan, J. J.; Tristram-Nagle, S.; Nagle, J. F. Phys. Rev. E 2009, 80 (021931), 021931-021912.

(39) Sun, Y.; Lee, C. C.; Hung, W. C.; Chen, F. Y.; Lee, M. T.; Huang, H. W. Biophys. J. 2008, 95, 2318-2324.

(40) Huang, H. W. Biophys. J. 1986, 50, 1061-1070.

(41) Harroun, T. A.; Heller, W. T.; Weiss, T. M.; Yang, L.; Huang, H. W. Biophys. J. 1999, 76, 3176-3185.

(42) Lundbaek, J. A. J. Gen. Physiol. 2008, 131, 421-429.

(43) Lundbaek, J. A. J. Phys.: Condens. Matter 2006, 18, S1305S1344.

(44) Lundbaek, J. A.; Collingwood, S. A.; Ingolfsson, H. I.; Kapoor, R.; Andersen, O. S. J. R. Soc. Interface 2010, 7, 373-395.

(45) Tristram-Nagle, S.; Kim, D. J.; Akhunzada, N.; Kucerka, N.; Mathai, J. C.; Katsaras, J.; Zeidel, M.; Nagle, J. F. Chem. Phys. Lipids 2010, 163, 630-637. 